\title{
Zwei Urkunden des koptischen Notars David, des Sohnes des Psate
}

\author{
Sebastian Richter (Leipzig)
}

Der koptische Tabellio David, Sohn des Psate, lebte im 8. Jh. n.Chr. in der Ortschaft Djême, die auf den Ruinen des Totentempels Ramses' III. in Medinet Habu entstanden war. ${ }^{1}$ Wir kennen ihn hauptsächlich durch seine Arbeiten, Tabellionenurkunden zur Beurkundung privatrechtlicher Angelegenheiten, die ihn uns während der dreißig Jahre zweier Indiktionszyklen, vom ersten Indiktionsjahr 733 (KRU 5) bis zum ersten Indiktionsjahr 763 (KRU 24), tätig zeigen. ${ }^{2}$ In Crums Publikation der Djêmepapyri ${ }^{3}$ ist David, S. d. Psate, als Schreiber von sechs Urkunden ausgewiesen: ${ }^{4}$ KRU 5, 19, 24 und 90 sind durch Davids Fertigungsvermerk autorisiert, die am Anfang und Ende zerstörte Urkunde KRU 98 und das Fragment KRU 102 sind von Crum wohl mittels paläographischer Evidenz dazugestellt worden. ${ }^{5}$ Im übrigen sind noch vier Zeugenunterschriften Davids belegt. ${ }^{6}$

${ }^{1}$ Cf. U. Hölscher, Medinet Habu Reports II. The Architectural Survey 1929/30, Univ. of Chicago Orient. Publ. N¹0, 1931, 50-56.

${ }^{2} \mathrm{Zu}$ den Datierungen cf. W.C. Till, Datierung und Prosopographie der koptischen Urkunden aus Theben, Wien 1962. Davids Vater Psate ist nach Davids Fertigungsvermerken nach 747 (KRU 5, Z. 99 Psate noch nicht als MdK, bezeichnet) gestorben. Wenigstens zu erwägen ist, ob jener Psate, S. d. David, Schreiber der beiden Urkunden KRU 85 und 107 und Zeuge in KRU 82, der Sohn von David, S. d. Psate ist. In Djême sind nämlich auch in koptischer Zeit Fälle der Kindesbenennung nach den Großeltern nicht selten, cf. G. Heuser, Die Personennamen der Kopten, Leipzig 1929, p. 9; Till, a.a.O., z.B. Geneaologie p. 149: Paham-Epiphanius-Paham; O. Medinet Habu 251 (ed. E. Stefanski/M. Lichtheim): Thomas-Biktor-Thomas u.ö. Auch ist Psate, S. d. David, deutlich jünger als David, S. d. Psate: Die von ihm gefertigten Urkunden sind beide 767/68 n.Chr. datiert (cf. Till, a.a.O., p. 31, 39), die von Psate mitbezeugte Urkunde KRU 107 ist auf einem Papyrus mit J. 155 H. $=771 / 772$ n.Chr. datierten arab. Protokoll geschrieben. Für den Fall der Verwandtschaft wüßten wir, da Psate sich in allen Fällen 'Sohn des seligen David' nennt, daß David, S. d. Psate, Zwischen Mitte des Jahres 763 (KRU 24) und 767/68 gestorben ist.

${ }^{3}$ W.E. Crum, Koptische Rechtsurkunden des achten Jahrhunderts aus Djême (Theben), Leipzig 1912 (= KRU); andere Editionen von Urkunden aus Theben enthalten keine Arbeiten von David, S. d. Psate; auch hat er nicht, gleich anderen routinierten Schreibern von Djême wie Aristophanes, S. d. Johannes, Johannes, S. d. Lazaros, Kyriakos, S. d. Petros und Psate, S. d. Pisrael, Steuerquittungen ausgestellt - was indessen nicht verwunderlich ist, da P.E. Kahles Folgerung gemäß die Quittungen der genannten Schreiber etwa zwischen 717 und 730 n.Chr. und die anderen (vor allem die anonymen - fast die Hälfte der inzwischen weit über 400 bekannten -) Steuerquittungen von etwa 710 n.Chr. an datieren (FS Äg. Museum Berlin, 1974, 283ff.).

${ }_{5}^{4}$ Cf. Crum, KRU, p. 462f., Index V.

5 Unter diesen sind KRU 5, 19 und 24 als Verkaufsurkunden stilisiert, sie werden (EГTPdфON $\mathrm{N}-)$ ПPגCIC $(5,6.14 .41 ; 19,24.79 ; 24,17.31 .102)$ und $\omega \mathrm{NH}(5,15 ; 24,115)$ genannt, wobei KRU 24 
Nun lassen sich durch Vergleich und Analyse dieser Texte weitere, textinterne Merkmale zur Charakterisierung ihres Schreibers gewinnen, nämlich Besonderheiten des Formulars und der Orthographie. Die Tabellionenurkunden des koptischen Notariats sind bekanntlich teils durch Übersetzung, teils durch Übernahme ganzer Klauseln den griechisch-byzantinischen Urkunden dieser Art nachgebildet worden. ${ }^{7}$ Nichtsdestoweniger ist in der Behandlung dieses Musters, in der Bevorzugung, Anordnung, Verknüpfung, Auffassung oder auch Verkennung vorgeprägter Wendungen ein 'persönlicher Urkundenstil' des Notars zu finden. Auch läßt sich ein individuelles Spektrum von 'dialectic misspellings' ${ }^{\prime}$ ermitteln und als Kriterium verwenden.

David, S. d. Psate, war ein geübter Urkundenschreiber. Seine Schrift ist eine flüssige, gut leserliche Kursive mit wenig Ligaturen. Seine Orthographie entspricht über weite Strecken makellosem Sahidisch. Dennoch finden sich teils sporadische, teils konsequent durchgehaltene Abweichungen vom literarischen Standard, die oft als typisch für den Dialekt von Theben erscheinen. ${ }^{9}$ Bei griechischen Ausdrücken, die fast die Hälfte des Wortschatzes von Davids Urkunden ausmachen, ${ }^{10}$ ist vor allem die Wiedergabe von Vokalen $(0$ und $\omega$ sind besonders anfällig für Verwechslung), nur in wenigen Fällen die Schreibung von Konsonan-

eine Erbteilung zum Gegenstand hat (zur Disposition des Geschäftes cf. A.A. Schiller, A Family Archive from Jeme, in: Studi in onore di Vincenzo Arangio-Ruiz, Vol. IV, Napoli 1952, p. 357361) und KRU 19, der Eigenart der beurkundeten Transaktion - der Übertragung eines Hauses durch einen Vater an seine Kinder als Ersatz für das in Notzeiten veräußerte mütterliche Erbe entsprechend, nur eine Auswahl von Formeln des Verkaufs aufweist. KRU 90, 98 und 102 sind Kindesschenkungsurkunden.

${ }^{6}$ KRU 43,75, 87,54, 95,41 und W.E. Crum, Varia Coptica, Aberdeen 1939, N 6, verso Z. 16.

7 Zur griechischen und koptischen Tabellionenurkunde cf. E. Sachers, Art. 'Tabellio' in RE IV A.2. 1932, Sp. 1847ff.; A. Steinwenter, Studien zu den koptischen Rechtsurkunden aus Oberägyp-

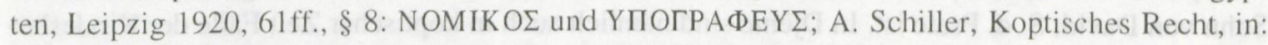
Krit. Vierteljahresschrift f. Gesetzg. u. Rechtsgesch. 25, 1932, 259, Steinwenter, Das Recht der koptischen Urkunden, HB der Altertumswiss. X,4,2, München 1955, 9-16.

${ }^{8}$ Dazu Crum, The Monastery of Epiphanius at Thebes, Vol. I, New York 1926, chapter X: The Language of the Texts, 232-256; Kahle, Bala'izah, London 1954, chapter VIII: Dialectical Variations in Sahidic non-literary Texts, 48-192; W.H. Worrell, Coptic Sounds, Ann Arbor 1934, Chapter III: Dialectic Misspellings in sixth- and seventh-century Theban Documents, p. 99ff. und P. Nagel, Der frühkoptische Dialekt von Theben, in: Koptologische Studien in der DDR, Wiss. Z. Univ. Halle, 1965, 30-45.

${ }^{9}$ Besonders markante 'Thebaismen' Davids sind der gelegentliche Gebrauch

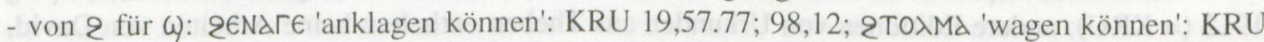

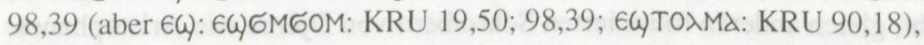

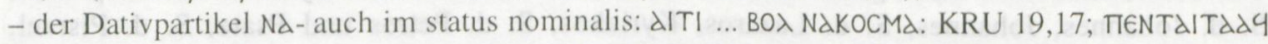

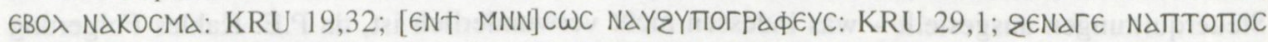

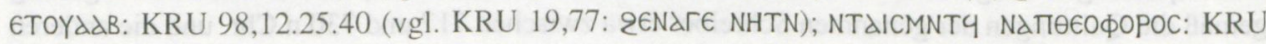
98,16 ,

- des Verbs BWK transitiv (Bedeutung 'senden' cf. Crum, CD 30a): dNBWK ₹ENPWM€ MחICTOC: 24,161 .

${ }^{10}$ David verwendet in den (einschließlich KRU 22 und 29) acht von ihm aufgesetzten Urkunden ca. 210 koptische und 190 griechische Lexeme. 
ten (typisch ist die Verwechslung von $\Delta$ und T) von orthographischer Regelwidrigkeit betroffen. Davids Urkunden zeichnen sich unter den Djêmepapyri durch klaren Duktus sowie durch die recht versierte Anwendung der aus dem byZantinischen Urkundenwesen überkommenen Klauseln aus. Es ist kennzeichnend, daß David die den griechischen Originalen näherstehenden, komplizierteren und mit griechischen termini technici stärker durchsetzten Varianten von Formeln bevorzugte und daß einige Wörter und Wendungen seines Repertoirs sonst nur noch in Urkunden des Aristophanes, S. d. Johannes, des Routiniers unter den Privatnotaren von Djême, zu finden sind.

Aufgrund der oben skizzierten Analyse können jetzt zwei weitere Urkunden aus dem Corpus der KRU als Arbeiten Davids reklamiert werden:

\section{KRU 22}

Diese Urkunde ${ }^{11}$ besteht aus zwei nachträglich zusammengeklebten Stücken, von Krall als 'Aa' und 'Ab' bezeichnet. Das Fragment 'Aa' enthält Reste des arabischen Protokolls und der ersten drei koptischen Zeilen mit der Anrufung der trinitarischen Personen und (unlesbarem) Datum. Zwischen diesem Blatt und dem Hauptstück 'Ab' fehlen vom protokollarischen Teil des Formulars die Intitulatio und die Adresse des Destinatärs (Papnouthios, S. d. Stephanos), vom Corpus der Urkunde Homologie, Willensfreiheitsklausel, Schwurformel, Verkaufserklärung und die Verschreibung des Kaufobjektes. Erst mit der Beschreibung der Lage des Kaufobjektes - eines um zwei Solidi feilen Hauses - setzt der Text wieder ein. Da sich im erhaltenen Teil der Urkunde der Aussteller nicht mehr selbst nennt, kennen wir seinen Namen nicht. KRU 22 nun partizipiert an folgenden orthographischen Eigenschaften der Urkunden Davids:

\section{$d d$ für $d$}

ETMMddY: KRU 5,53; 19,20,45,49/50,56; 22,43; 24,46/47,51,57,58,65,106/7; ein einziges (freilich unsicheres) Mal bei David €TMMdY: 29,25. In den KRU sonst z.B. 37,68 (dort auch NMddY, 2ddT); 122,40 (dort auch $\Pi € \varangle d d=$ ); 106,83,127/28 (aber vom selben Schreiber Chmntsnêu, S. d. Schenute, $\in$ TMMAY: 12,25,27,32,39; 13,54). Überhaupt ist in den nichtliterarischen Texten der thebanischen Fundorte die Doppelschreibung von $₫$ häufiger als andernorts. ${ }^{12}$

11 Österreichische Nationalbibliothek K 10992; zuerst ediert und übersetzt von J. Krall, Zwei koptische Verkaufsurkunden, WZKM 2, 1888, p. 35-36; neu ediert und übersetzt von W.C. Till, Die koptischen Rechtsurkunden der Papyrussammlung der Österreichischen Nationalbibliothek, Wien 1958 = CPR IV, $N^{\circ} 26$, p. 25-27 und Tafel II, 5 (Photographie Z. 42-56); vollständige Photographie bei W. Brunsch, Kleine Chrestomathie nichtliterarischer koptischer Texte, Wiesbaden 1987, Tafeln 10a-10e; zum arab. Protokoll cf. A. Grohmann, Allgemeine Einführung in die arabischen Papyri, Wien 1923 (= CPR III,1.2), N 125, p. 114f. und Tafel 26d; zur Datierung cf. Till, Datierung und Prosopographie (Anm. 2.): arab. Protokoll J. 142 der Hidschra $=759 / 760$, also bald nach 759/760; eine der späteren Urkunden des David, S. d. Psate.

12 P.E. Kahle, Bala'izah. Coptic Texts from Deir el-Bala'izah in Upper Egypt, London 1954, Vol. 1, p. 61, (\$ 11); Crum, The Monastery of Epiphanius, Vol. I (Anm. 8), p. 242. 
$\epsilon$ für d

ЄХN für dðN: KRU 5,57; 22,49; 24,114. Die Formel $d \chi N N d I \Delta \epsilon$ THPOY... verwendet in diesem Wortlaut sonst nur Aristophanes, S. d. Johannes: Dieser schreibt fünfmal korrekt $\Delta \searrow \mathrm{N}$ : 11,$56 ; 14,83 ; 17,42 ; 48,52 ; 87,36 / 37$, zweimal aber $\in \searrow N$ : 35,$87 ; 41,95 / 96$. $€ \triangle N$ 'ohne' in den KRU außerdem 67,140 und 106,155. Generell ist der Wechsel von $₫$ zu $\in$ in den thebanischen Texten sehr häufig. ${ }^{13}$

\section{$€$ für Silbenstrich}

stets PMENHI: KRU 5,46; 19,54; 22,32; 24,94 (So, nicht חPMNENHIsic wie in KRU); 90,$3 ; 98,12,28$. Der Wechsel zwischen $€$ und Silbenstrich ist in Theben nicht häufig. ${ }^{14}$

\section{H für Silbenstrich}

2d2THN: KRU 22,37,40; 24,100,104, aber 2d2TN: 5,50.

Dieser Wechsel ist in den thebanischen Texten sehr selten (2ג2THN in den KRU aber z.B. auch 56,8), nördlich von Theben - besonders in Aschmunein und Deir el-Bala'izah - üblich vor $M, N$ und P. ${ }^{15}$

\section{$\epsilon I$ für $\epsilon$}

MNNEINEPHY: KRU 5,32; 22,9; 24,163, nie MNNENEPHY; Poss.-Art. aber sonst stets NEN-. MNNEINEPHY ist in den KRU nur hier belegt (5,32 zitiert bei Kahle, a.a.O., 72); die Schreibung (€)I statt $€$ ist aber auch sonst in Theben bekannt, ${ }^{16} \mathrm{z} . \mathrm{B}$. KRU 3: K[€]I- für K€- Z. 4; ЄI- für Präp. Є-Z. 47f.; KRU 92,38: ЄINOP€K'wir schwören'; unpubliziertes Ostrakon, Ägyptisches Museum der Universität Leipzig Inv.-Nr. 7245, Z. 10.15: MAPEI- (Basis des kausativen Imperativs vor nominalem Subjekt).

\section{0 für $\omega$}

Statt $\omega \omega \Pi$ stets $\omega$ )

Außer KRU 1,28 u.ö., 2,18, 4,28 u.ö. (alle drei von Schenute, S. d. Chmtsnêu geschrieben) und 6,23, die jeweils woom als status absolutus des Verbs bieten, ist in den KRU ausschließlich $\omega \omega \Pi$ belegt. Generell ist der Wechsel von $\omega$ zu O (vice versa) in nichtliterarischen Texten häufig. ${ }^{17}$

13 Kahle (Anm.12), 59 (§ 7); Crum (Anm. 8), 237.

14 Kahle (Anm.12), 59f. ( $\$ 8)$; Crum (Anm. 8), 238.

15 Kahle (Anm.12), 54 (§ 2).

16 Kahle (Anm.12), 72 (§ 23); Crum (Anm. 8), 239.

17 Kahle (Anm.12), 90 (§ 61). 
Präp. N vor Labial nicht zu M assimiliert ${ }^{18}$

NTBO $\lambda$ : KRU 5,47,48,49; 19,58,60; 22,34 (2x),35; 24,96,97,98; 90,4,5; 98,13,14; nie MПBO入.

NTZI(ГOC): KRU 22,10, aber MாZI: 5,33.

Schreibung griechischer Wörter

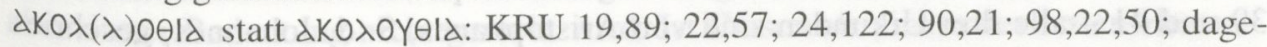

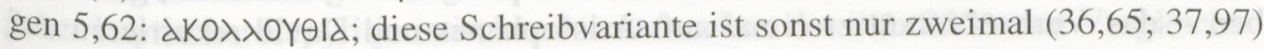
in den KRU belegt.

dPXWNTIKWN statt dPXONTIKWN: KRU 5,52; 22,41/42; 24,105; [90,18]; 98,15; in dieser Schreibung sonst nur zweimal $(92,35 ; 107,20 / 21)$ in den KRU belegt.

EKK入HCIdCAIKON: mit $\Delta$ statt T nur in KRU $22,42,24,105 / 6,90,18$ und 98,15 belegt.

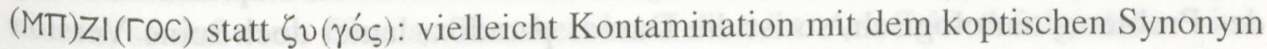
MTG)I, nur in KRU 5,33 und 22,10 belegt.

MథdNIZ€ statt ЄMфdNIZ€: KRU 5,60; 19,82; 22,17,53; 24,118; [29,20,30]; 90,19; 98,45; auch sonst sehr oft in den KRU.

¿WPIZ€ statt 2OPIZ€: KRU 5,56; 22,48; 24,113; auch sonst mitunter in den KRU.

ПРОтОтүП 24, $107 / 8$ belegt.

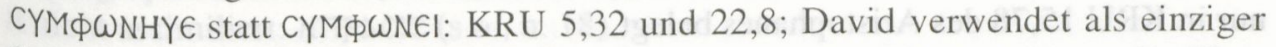
Schreiber in den KRU die Form сүмфWNHY€, als sei das Verb kein contractum, sondern vom Flexionstyp ' $\pi \propto \iota \delta \varepsilon v \dot{\omega} \omega$ '.

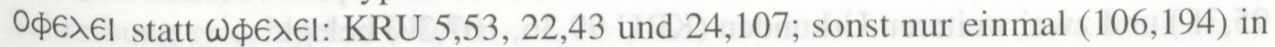
den KRU belegt.

Darüber hinaus zeigt KRU 22 signifikante Merkmale des Formulars der Urkunden Davids:

Formel über den Kaufpreis

Die Wendung '(Der Kaufpreis) NTANCYMфWNHY€ EPOC MNNEIN€PHY' in KRU 5,32 und 22,8f. enthält gleich zwei in den KRU nur hier belegte Idiosynkrasien (s.o.).

Formel über den Eigentumserwerb

Auf die Formel $\searrow$ INTENOY $\Delta €$ GPOK NTOK ... folgt (ausschließlich) in KRU 5,35ff., $22,15 \mathrm{ff}$. und 24,74ff. nicht die Übereignungsformel mit dem dafür konstitutiven Futur II $\epsilon=N d \epsilon I \epsilon 20 Y N$ und den es fortsetzenden Konjunktiven von Verben der Besitzergreifung, sondern unmittelbar die Aufzählung der Eigentumsrechte. Vermutlich hat David die Formel so umgebildet, weil nach seinem Formular bereits zwischen Verschreibung des Kaufobjektes und dessen Lagebeschreibung die

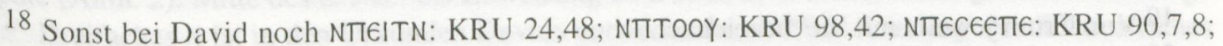
98,6/7,18; \NNTOOY (aus ХMTOOY korrigiert!): KRU 24,45; NாPגCIC: KRU 19,79/80, 24,17; 29,4

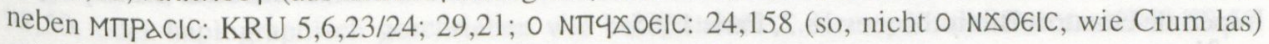
neben Mா4メO€IC: KRU 24,69; 29,26; NT€NTdITdd4: KRU 19,31. 


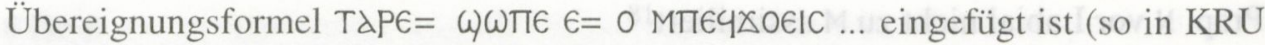
5, 24 und 29 (s.u.), dieser Passus ist in KRU 22 nicht erhalten.

Nichtangriffsklausel

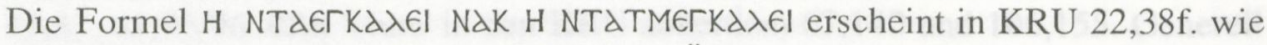
(ausschließlich) in KRU 5,51 und 24,101f. Überhaupt weisen sonst nur KRU 15, 39 und 41 - alle drei Urkunden sind von Aristophanes, S. d. Johannes, geschrieben - das Verb $\epsilon \Gamma K d \lambda \in I$ zweimal auf. Aristophanes und David rekurrieren auf dieselbe griechische Formel, hatten aber offenbar beide keinen Begriff vom Sinn des zweifachen $\epsilon\ulcorner K d \lambda \epsilon \mathrm{I}$ : Während Aristophanes einmal (KRU 39,59ff.) wenigstens das Objekt variiert: '... oder dich anklagt oder deine Kinder anklagt ...', läßt er es die anderen Male (KRU 15,77f., 41,84f.) mit der scheinbaren Dittographie ${ }^{19}$ '... oder dich anklagt oder (dich) anklagt ...' bewenden. Ist Aristophanes' Lösung des Problems tautologisch, so ist die Davids paradox, lautet doch seine Version der Formel: '... oder daß ich dich anklagte oder daß ich dich nicht anklagte ...'. Nur einem von beiden kann natürlich bei Strafe abgeschworen werden. In der griechischen Vorlage wird, soweit ich sehe, dagegen im Tempus variiert, wenn der Aussteller sich verpflichtet, daß er weder anklagt, noch anklagen wird: $\mu \eta \dot{\tau} \tau$

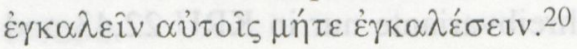

Die Formel NTAKIM $€$ POK 2ג2THN $\lambda d d y$ N- ist außer in KRU 22,40 und 24,103 nur in KRU 15,78 des Aristophanes belegt.

Die Opposition $\triangle$ PXONTIKWN / EKKXHCIdCTIKWN ist außer in Davids Urkunden KRU 5, 22, 24, 90 und 98 noch in zwei Urkunden des Aristophanes (KRU 48,44f., 95,3f.) und zwei weiteren Urkunden (KRU 99,36, 107,20) belegt.

\section{Strafklauseln}

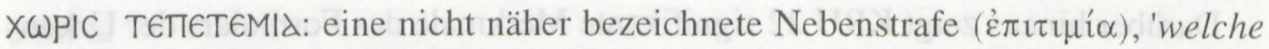
die Gesetze über den verhängen, der übertritt', wohl eine den Eidbrüchigen als solchen treffende Sanktion, ${ }^{21}$ ist außer in den Urkunden Davids KRU 5, 22, 24 und denen des Aristophanes KRU 10, 11, 14, 15, 17, 41, 48 noch in KRU 6, 28, 82 und 99 belegt.

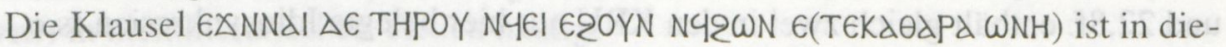
sem Wortlaut außer in KRU 5, 22 und 24 nur von Aristophanes in KRU 10, 11,

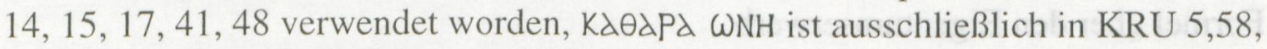

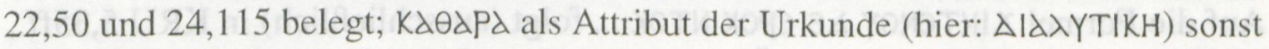
nur in KRU 48,54 des Aristophanes.

Für KRU 22 läßt sich schließlich paläographisch der Beweis für die Urheberschaft Davids, des Sohnes des Psate, und damit eine Bestätigung der textkritischen

19 Von Crum jeweils mit 'sic' bezeichnet.

20 P.Paris 20,19, ed. W. Brunet DePresle, Notices et extraits des manuscrits de la Bibliothèque Impériale et autres Bibliothèques 18.2, Paris 1865.

${ }^{21}$ Cf. A. Steinwenter, Das Recht der koptischen Urkunden, München 1955, p. 14, n. 3. 
Argumente erbringen. ${ }^{22}$ Insofern unterscheidet sich dieser Fall von jenem der zweiten Urkunde:

\section{KRU 29}

Da der Originalpapyrus ${ }^{23}$ schon zur Zeit von Crums Edition der Djêmepapyri nicht mehr auffindbar war, ist die Textgrundlage für KRU 29 Ciascas Edition, die aber dieser Bedeutung nicht gerecht wird. Ist Ciasca als einem der ersten Herausgeber koptischer Papyri juristischen Inhaltes schon des ihm fehlenden Vergleichsmaterials halber zuzubilligen, daß er den in flüssiger Urkundenschrift beschriebenen und seinerzeit offenbar schon stark zerstörten Papyrus nicht richtig zu lesen vermochte, so hätte er wenigstens die Originalzeilen markieren und die Größe der Textlücken genauer angeben können. Crum nun hat in KRU seine Ergänzungen und seine nur zum Teil wahrscheinlichen Emendationen nicht konsequent ausgewiesen, so daß stets Ciascas Text heranzuziehen ist. Am Schluß folgt daher eine Neuedition des Papyrus auf der Grundlage des von Ciasca a.a.O. gebotenen Textes.

KRU 29 partizipiert an folgenden orthographischen Eigenschaften der Urkunden Davids:

WOח: nur in KRU 5, 22, 24 und 29 in dieser Schreibung, s.o.

Haplographie 2NNOMOC (statt 2NNNOMOC) in KRU 29,5 wie 5,7 und 24,19.24

$\mathrm{N}$ nicht assimiliert: NMPdCIC (s.o., n. 18).

Dativ Nd-im status nominalis: NגY2YחOГPגф€YC (s.o., n. 9).

\section{Schreibung griechischer Wörter}

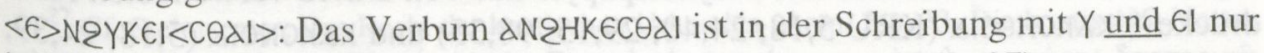
in KRU 5,28, 24,70 und 29,27 belegt; s.u. (Kommentar zu Zeile 27).

$\Pi$ POथ૯PHCIC statt ПP02dIPECIC: Diese Schreibvariante des in den Willensfreiheitsklauseln der KRU häufig verwendeten Wortes ist ausschließlich in KRU 5,17, 24,38, 29,14/15, 90,16 und 98,35 belegt, s.u. (Kommentar zu Z. 16).

22 Tatsächlich wurden mir erst im Anschluß an die sprachliche Analyse die beiden publizierten Photographien (s.o. n. 11) dieser Urkunde bekannt; übrigens sind sonst m.W. keine Photographien von Urkunden Davids publiziert, zu vergleichen ist lediglich ein Faksimile von KRU 98,26-34 bei E. Revillout, Actes et constrats des Musées Égyptiens de Boulaq et du Louvre, $1^{\text {er }}$ Fascicule: Texts et Fac-Simile, = Études Égyptologiques, $5^{\text {ième }}$ Livraison, Paris 1876, Pl. 11; an dieser Stelle sei Frau Dr. Ingeborg Müller, Papyrussammlung Berlin, für die Zusendung einer Photographie von KRU $24=$ P.Berlin 10606 gedankt.

23 Vormals Bibliotheca Vaticana, zuerst ediert 1881 von A. Ciasca, I papiri copti del museo Borgiano, papiro originale $\mathrm{N}^{\circ} 2$, testo copto VII. p. $\overline{\mathrm{K}} \Gamma \mathrm{f}$.; Übersetzung bei Till, Die koptischen Rechtsurkunden aus Theben, Wien 1964, p. 128f.; Datierung nach Till, Datierung und Prosopographie (Anm. 2): Mitte des 8. Jhs.; die Zuweisung an David, S. d. Psate, bestätigt Tills Datierung.

${ }^{24}$ Dreifaches $\mathrm{N}$ ist in den Urkunden Davids entweder durch 'Vokalisierung' des Silbenstriches aufgespalten, z.B. beim Genitiv Plural NNNOMOC = NENNOMOC: KRU 5,63, 19,89, 24,122, 90,21, 98,50 , oder haplographisch geschrieben wie bei 2 NNOMOC, aber auch <N $>$ NOMOC: KRU 22,57 (Genitiv Plural), 5,23f., 29,21 (Präp. N-, s.u., n. 28). 


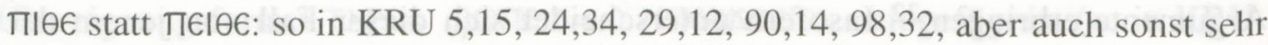
häufig in den KRU und überhaupt in thebanischen Texten belegt.

XPICTHPION statt XPHCTHPION: diese Schreibvariante ist außer in KRU 5,28, 24,70 und 29,27 nur ein weiteres Mal $(27,33)$ in den KRU belegt.

KRU 29 weist folgende signifikante Merkmale des Formulars der Urkunden Davids auf:

\section{Intitulatio}

ЄТРЕЧ२ҮпоГРАфє 2АРОүsic: Eine Eigentümlichkeit in Davids Urkunden ist diese, daß die Aussteller hier nicht dem subjektiven Stil des Cheirographon gemäß durch das Personalpronomen der ersten Person, sondern in allen drei Belegen (KRU 5,5, 24,25, 29,3) durch das der dritten Person (2גPoY/2৯PO4) repräsentiert werden. Diese Nachlässigkeit ist sonst nicht in den KRU belegt. 25

\section{Homologie}

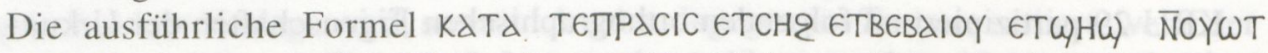
2ITOOTC ist sonst in den KRU außer von Davids in den Urkunden KRU 5, 24 und 29 nur von den beiden Schreibern Aristophanes, S. d. Johannes (KRU 11, 14, 15, 27), und Chmtsnêu, S. d. Sinouthios (KRU 13), verwendet worden.

Willensfreiheitsklausel

KRU 29,12ff. stimmt wörtlich mit KRU 5,15ff., 24,33ff. und 98,32ff. überein: Signifikant sind dabei die in den Djêmepapyri nur von David verwendeten Ausdrücke ${ }^{26}$ KdKOHӨEId (KRU 5,18; 24,40; 29,$\left.15 ; 98,35\right)$ und KdKONOId $(5,18 ; 24,39$; $29,15 ; 98,35 / 36)$ sowie die griechische Formel 'in jedem schönen [und guten] Vor-

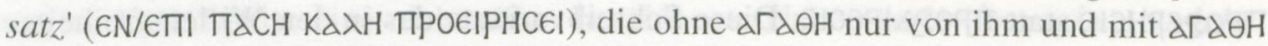
in den KRU noch von den drei Schreibern Aristophanes, S. d. Johannes (KRU 11, 14, 15, 87), Senouthios, S. d. Chmtsnêu (KRU 1, 2, 4), und Psate, S. d. David (KRU 85), geschrieben worden ist.

\section{Schwurformel}

KRU 29,16-18 stimmt wörtlich und buchstäblich mit KRU 5,19-21, 24,41-45 und 98,37-39 überein.

25 In KRU 69 bezieht sich 2dPOY eindeutig auf die schreibunkundigen Zeugen, denen ein Schreibhelfer (P€YC2dI) beisteht; in KRU 74 und 118 ist jeweils die ganze Textpassage objektiv stilisiert.

26 Sie sind in den Text der Formel elliptisch eingefügt:

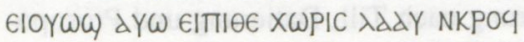

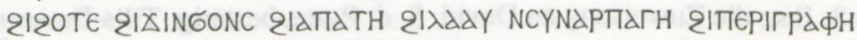

EMNOYANATTH NOYWT KH NAI €QPAI

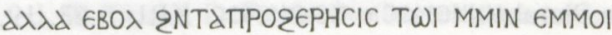

2IKAKONOId NIM IIKAKOHEEId

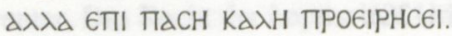


Verkaufserklärung und Verschreibung des Kaufobjektes

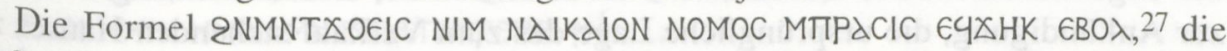
außer in Davids Urkunden KRU 5 und 29 noch in fünf Urkunden des Aristophanes (KRU 10, 11, 14, 15 und 27) und drei weitere Male in den KRU (4, 7 und 28) belegt ist, weist in KRU 29 wie in KRU 5 (sonst nur in KRU 15) die Haplographie $\triangle I K \Delta I O N<N>$ NOMOC auf. ${ }^{28}$

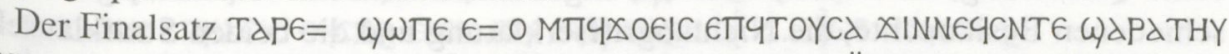
MNNEYXPICTHPION THPOY ETENQYKEICOAI EPOOY ist eine Übereignungsformel und steht innerhalb der Verkaufsurkunden eigentlich im Kontext der Klausel über den Eigentumserwerb, unmittelbar hinter den Formeln über den Kaufpreis. Zwischen die Verschreibung des Kaufobjektes und dessen Lagebeschreibung vorgezogen, erscheint diese Formel in Davids Urkunden KRU 5,26ff., 24,68ff. und 29,26ff. sowie in KRU 2,21 (von Senouthios, S. d. Chmtsnêu), 15,38 und 27,31 (beide von Aristophanes, S. d. Johannes). ${ }^{29}$

\section{Bemerkungen zu Inhalt und Formular von KRU 29}

Vom Formular der Urkunde haben sich Anfang und Ende erhalten: Der Text beginnt mit der Intitulation der Urkundenaussteller. Zu der nachfolgenden Homologie - die Urkunde hatte wohl wie KRU 5 keine Adresse des Destinatärs gehören Reste von Namen und die Formel $<K d T d T>\epsilon \Pi P d C I C \epsilon<T>C H 2 \epsilon<T>-$ $B \in B d \mid O Y \in<T>G) H G$ ) etc. auf $Z$. 11. Es folgen, fast unversehrt, Willensfreiheitsklausel und Schwurformel. Mit der Erklärung, verkaufen zu wollen, beginnt auf Z. 18

27 Cf. Steinwenter, Das Recht der koptischen Urkunden (Anm. 21), 33: Die koptischen Notare

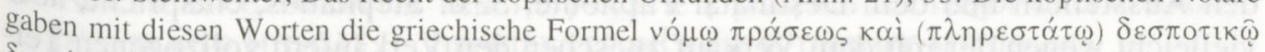
$\delta_{\text {Iאaí }}$ (wie in den byzantinischen P.Mon. 4,$8 ; 11,14$ ) in koptischer Sprache wieder.

${ }^{28}$ Für die Analyse dieser koptischen Formel ist die am häufigsten belegte Gestalt der Formel

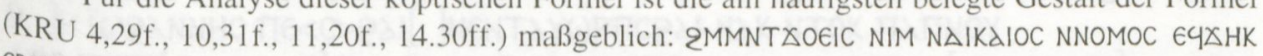
EBOX.

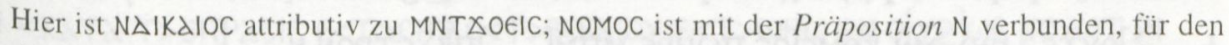
Genitiv müßte NOMOC, und sei es unbestimmt, determiniert sein. MTPdCIC ist nun wieder attributiv ZU NOMOC. $€ 4 X H K \in B O \lambda$ kann sich nur auf NOMOC, das einzige männliche Nomen, beziehen und ist wegen dessen Indeterminiertheit als 'unechter Relativsatz' formuliert. Wörtlich ist also zu übersetzen: 'In jeder gerechten Herrschaft nach vollständigem Verkaufsgesetz'.

ET $\triangle H K$ in KRU 27,22f. ist dementsprechend als Verschreibung (oder Verlesung?) zu betrachten, wie ja auch alle anderen Urkunden des Aristophanes $€ 4 \times H K$ haben. Ist, wie in den Urkunden KRU 7,19f. des Priesters Senouthios und KRU 28,4 des im griechischen ziemlich bewanderten Kyriakos, der Umstandssatz vor NNOMOC MTPACIC gezogen, so muß er sich auf MNTðO€IC NIM beziehen und steht folgerichtig beide Male im Plural ( $€ Y \searrow H K \in B O \lambda)$. Hier wurde also $\pi \lambda \eta p \varepsilon \sigma \tau \alpha \dot{\tau} \tau \omega$ nicht auf $v o ́ \mu \omega \pi \rho \alpha ́ \sigma \varepsilon \omega \varsigma$, sondern auf $\delta \varepsilon \sigma \pi \circ \tau \iota \kappa \hat{~} \operatorname{cozogen}$. Derselbe Plural, aber nach NNOMOC MMPdCIC in KRU 5,23f., ist entweder vom Schreiber David oder vom Editor Crum fälschlich gebraucht worden: Vermutlich letzteres, da KRU 29,21 die richtige Form aufweist und 4 und $Y$ in Davids Kursive einander stark ähneln. Die einmal bei Aristophanes (KRU 15,28ff.) und beide Male bei David $(5,23 \mathrm{f}$., 29,21$)$ belegte Form NOMOC statt NNOMOC ist eine Haplographie, die sicherlich durch das vorhergehende Wort $\triangle I K \Delta I O N$ mitverursacht wurde (s.o. n. 24).

${ }^{29}$ In gleicher Funktion und Stellung erscheint die Wendung $X \in K \Delta C$ EKN $\Delta K \in \lambda \in Y \in$ MMOOY in KRU 3,23. 
der engere Kontext des Geschäftes. Nun setzt sich der Zusammenhang noch bis zur Ankündigung, die ursprüngliche Lage des zum Verkauf stehenden Hauses zu zeigen, fort. Die Lücke, die nach Crums Aufteilung des Textes Zeile 29 unterbricht, muß etwa die halbe Urkunde enthalten haben: hier fehlen nämlich nach der Nennung der Anrainer die Formel über den zuvor ausgehandelten Kaufpreis und seine Quittierung, die Klausel über den Eigentumserwerb mit Aufzählung der verschiedenen Besitzrechte, die Nichtangriffserklärung und die beiden Strafklauseln. Vom Urkundenschluß sind dann wieder Teile der Formel über die Urkundenerrichtung sowie die Stipulationsklausel vorhanden. Absolution, Zeugenunterschriften und Kompletionsvermerk fehlen spurlos. Crums Emendation $\langle\Delta\rangle \mathrm{H} \lambda \mathrm{ON}$ OTlauf Z. 31 läßt die noch folgenden Schriftreste als einen Zusatz zur Urkunde erscheinen.

Leider ist weder in der Intitulatio noch in der Homologie der Name eines Ausstellers vollständig überliefert, wir kennen entweder nur den Vornamen (Komeios Z. 1) oder nur die Filiation (Anna Z. 1; Geôrgios Z. 8) oder den Namen des Ehemannes einer Mitausstellerin (Johannes, Z. 9). Möglicherweise ist der Z. 23/24 $\Pi € N M \Delta K,<N>€ I \omega T$ 'unser seliger Vater' genannte Ammonius der Großvater der Aussteller und 'unsere selige Mutter' (Z. 24, 28) Johanna seine Tochter. ${ }^{30}$ Diese zur Zeit der Urkundenerrichtung verstorbenen Vorfahren jedenfalls haben bewegliche und unbewegliche Güter hinterlassen, welche von den Ausstellern, einer Erbengemeinschaft, an Papnoute, den Sohn des Stephanos verkauft werden. Hauptobjekt ist ein Haus, das 'südlich der Gemeindekirche' von Djême lag. In Z. 29/30 wird die Urkunde Teilungs- und Auseinandersetzungsurkunde genannt, termini technici einer Disposition, die, soweit man sieht, nicht dem Verhältnis zwischen den Ausstellern und dem Destinatär Papnoute, S. d. Stephanos, entspricht, aber vielleicht wegen der mit dem Verkauf zwischen den Verkäufern vollzogenen Teilung des gemeinsamen Erbes gewählt wurde.

\section{Ciasca, I papiri copti, testo copto VII, Aufteilung der Zeilen nach KRU 29}

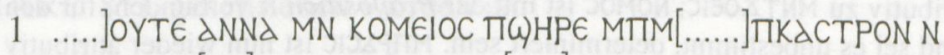

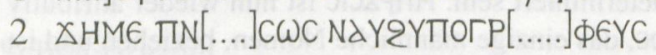

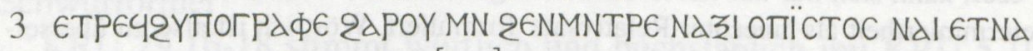

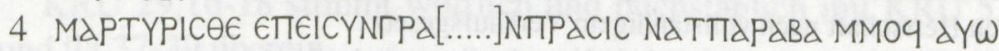

\section{Ergänzter und emendierter Text, Unterstrichenes schon von Crum ergänzt}

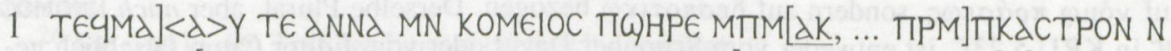

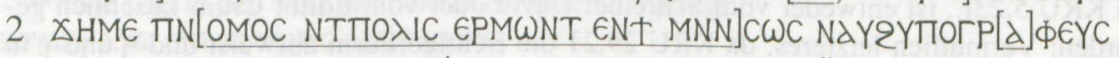

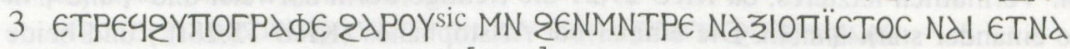

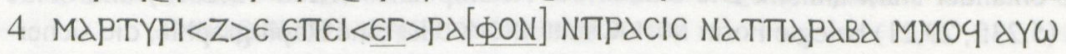

${ }^{30}$ Cf. die Emendation von Z. 24 und den Kommentar dazu. 


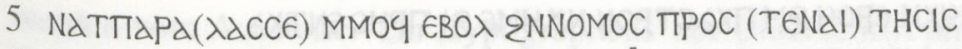

6 TWN MMIN MMON MN TENETITPETE $+[\ldots \ldots$.

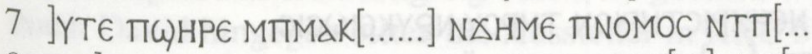

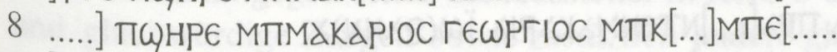

9 ]

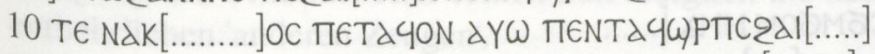

11 2YKaION €TPACIC EYCH2 GBEBdIOY EYG)HG) QN O[........]

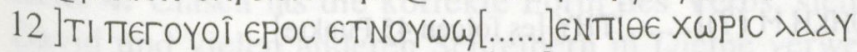

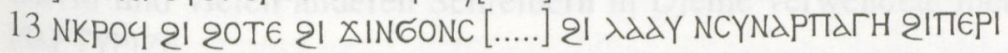

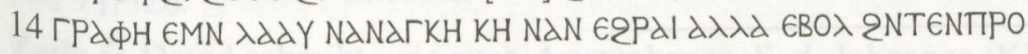

15 2૯PHCIC TWN MMIN EMMON 21 KAKONOId NIM 21 KAKOHӨEId

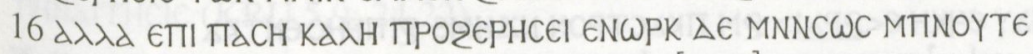

17 MANTOKPATWP MN TOYХAI NNENХICOOYE[.......]EХWN TENOY

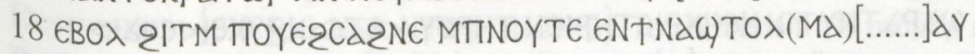

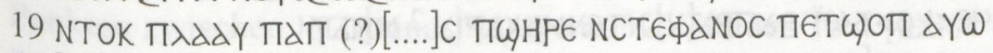

20 [...] ENTOTOC (NIM EYNAM)фaNIZE M(MOY) N(2H)TEY 2N

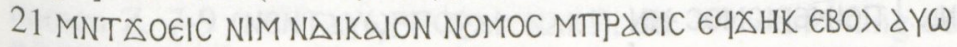

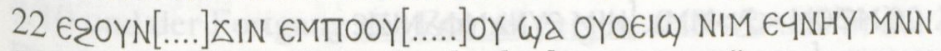

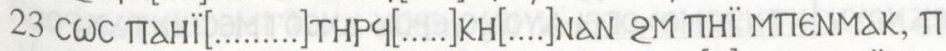

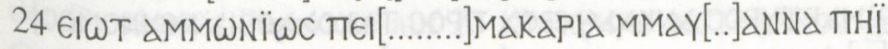

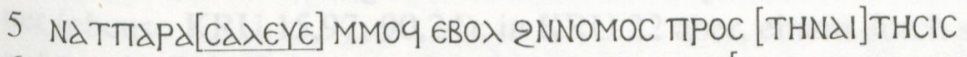

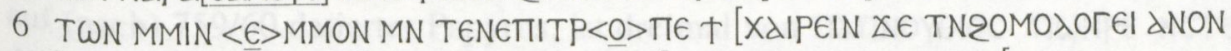

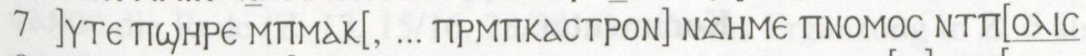

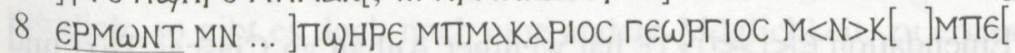

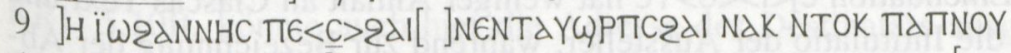

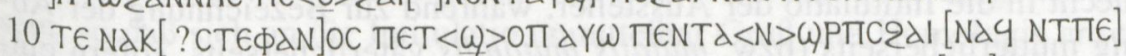

$11<K d T d T>\epsilon \Pi P d C I C \in<T>C H 2 \epsilon<T>B \in B d I O Y \in<T>\omega H G)$ 2N O[Y2]

$12[$ TOOTC $d N] T I \Pi \epsilon<-N>0 Y O I ̈ ~ \in P O C \in\{T\} N O Y \omega \omega)[a Y \omega] \in N \Pi I \theta \epsilon X \omega P I C \lambda d d Y$

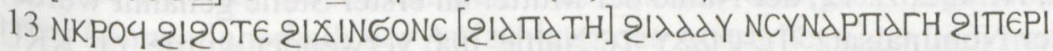

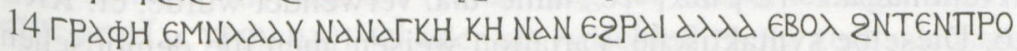

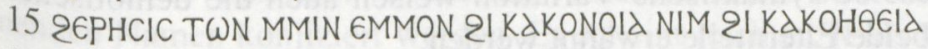

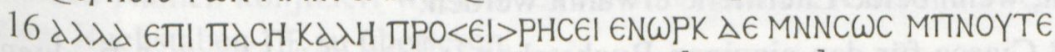

17 ПגNTOKPATWP MN TOYXdI NNENXICOOYE [ETAPXEI] EXWN TENOY

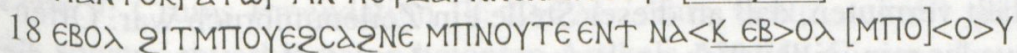

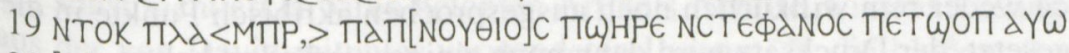

$20[\Pi] \in N<T \Delta N>C<Y M>\phi \Delta N I Z \in M<\Pi>\epsilon 4<P d N>2 N$

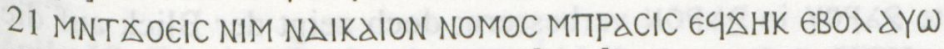

$22<\in N C>2<\Delta I>N[\Delta K] \Delta I N \in M T O O Y$ [N20]OY G)dOYOEIG) NIM EYNHY MNN

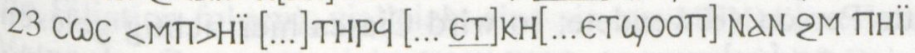

$M \Pi \in N M A K,<$ N $>$

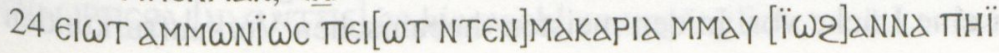




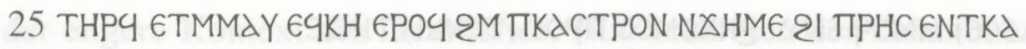

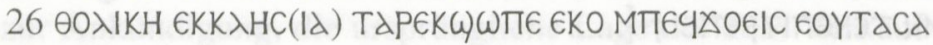

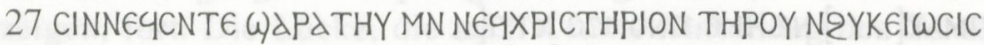

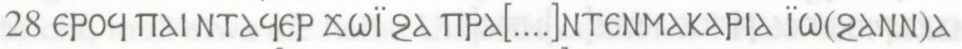

29 MМגY ПРОс $\theta \in[$.... .] $\Pi \epsilon I M E P I C M O C ~ M N$

$30 \Delta I d \lambda Y(\mathrm{ClC})[\ldots ..] \operatorname{cop} \triangle \mathrm{C \sigma} \mathrm{M \sigma OMMMd}[\ldots \ldots$.

$31]$ NCEXNOYN[........] dH HON TI[....]

32 NPח $[. ..] \chi N \in X \omega I[\ldots .] .0 \Theta H K H M(?)[\ldots . .] T H$.$Y [es folgen 45$ Punkte]

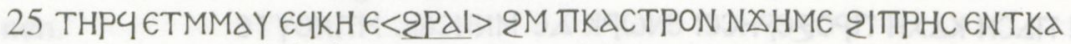

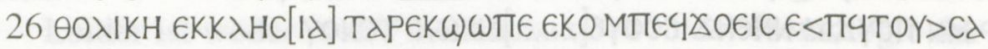

$27<\chi>$ INNEYCNTE ()גPATHY MN NEYXPICTHPION THPOY <ETE $>$ N2YKEI<CEd।>

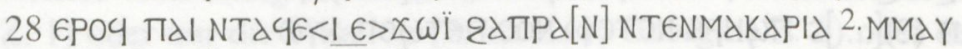

29 1.

TETPATWNON...] П ПEIMEPICMOC MN

$30 \Delta I d \lambda Y[\mathrm{CIC}]$ COPХ C6MGOM <2>MMd [NIM EYNAM

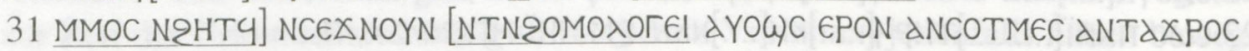

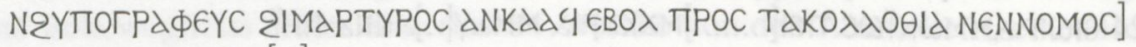

$<\Delta>\mathrm{H} \lambda \mathrm{ON}<\mathrm{O}>\mathrm{TI}[]$

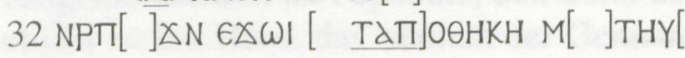

\section{Kommentar}

Zeile 1: Crums Emendation $\epsilon]<1><0>T \in$ hat weniger Anhalt an Ciascas Text und paßt schlecht in die Intitulatio der Aussteller, während zur Bezeichnung der Abstammung mütterlicherseits (bzw. mutatis mutandis väterlicherseits, sofern einmal, wie z.B. Crum, Catalogue of the Coptic Manuscripts in the British Museum, London 1905, $\mathrm{N}^{\circ}$ 452, Z. 12, der Name der Mutter an erster Stelle genannt wurde) regelmäßig der Nominalsatz 'TE4MAdY T€ Name' u.ä. verwendet wurde, cf. KRU 24,$25 ; 35,12$ u.ö. Dieselbe syntaktische Variation weisen auch die demotischen Urkunden auf, wenn beide Elternteile erwähnt werden.

Zeile 2: Daß Ciasca für den einzigen Buchstaben [ $d$ ] eine Lücke von 5 Punkten veranschlagte, läßt vermuten, daß an dieser Stelle ein Zeilenumbruch war. Offenbar hat Ciasca weder rein willkürlich noch ausgesprochen akribisch Punkte in die Textlücken gesetzt, der Drucksatz wird dann noch einiges verändert haben.

Zeile 3: Crum wollte 2גPOY in 2גPON verbessern; doch zeigt der Blick auf die Parallelstellen in den KRU, daß gerade David (KRU 5,5; 24,15) an dieser Stelle des Formulars die dritte Person verwendete; so wird Ciasca hier richtig gelesen haben. Crums Emendation $2 \in N[K \in] M N T P E$ ist weder ad sensum geboten, noch sieht Ciascas Text hier eine Lücke vor. In den meisten thebanischen Verkaufsurkunden 
werden vom Aussteller 'ein Hypographeus' zur Unterschrift und 'andere glaubwürdige Zeugen' zur Bekräftigung (TגメPO) gebeten (KRU 1, 2, 3, 4, 6, 7, 10 u.ö.). Nach David hingegen bitten die Aussteller in KRU 5 und 24 'einen Hypographeus' und 'glaubwürdige Zeugen' sowie zusätzlich 'andere glaubwürdige Zeugen' zur Befestigung der Urkunde. Hier fehlen nun lediglich die nach diesem Formular leicht entbehrlichen 'anderen Zeugen'.

Zeile 4: Ciasca las die korrekte Form des Verbs, sicher stand aber hier die von David und vielen anderen Schreibern in Djême verwendete metathetische Form MAPTYPIZE.

Zeile 5: David schreibt, wie auch Aristophanes, S. d. Johannes (KRU 10,9), ПPOC THNAITHCIC (KRU 5,9; 24,22).

Zeile 6/7: Crum ergänzte die Lücke bis ]YT€ (Z. 7 Anfang) mit der Adresse des Destinatärs: [€NC2dI NaK NTOK $\Pi \Delta \Pi N O]$. In diesem Fall müßte in der Lücke von 8

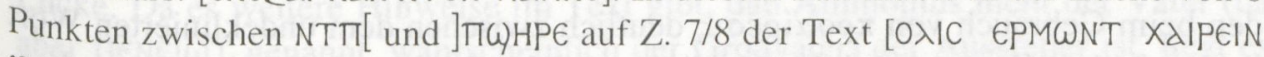
$\triangle \in$ TN20M0入OTEI גNON Name] gestanden haben, da die Folge von Ausstellernamen Z. 7-9 (NENTAYGPTC2גI NגK!), die Wiederholung des Destinatärs auf Z. 9/10 und der Fortgang der Formel zeigen, daß wir uns in der Homologie befinden. Das ist recht viel Text, allein wir kennen nicht die Größe der Lücken. Nun wird der Destinatär Papnoute auf Z. 19 ПఢHP€ NCT€ФANOC genannt, während der von

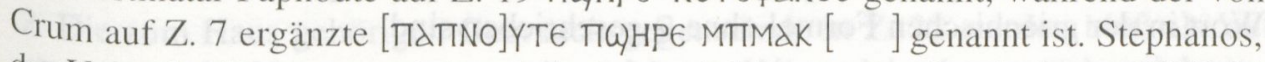
der Vater des Papnoute, wird auch in der nach Märtyrerära fest datierten KRU 14 (756 n.Chr.) in der Zeugenunterschrift des Papnoute Z. 94 als MdKdPIOC bezeichnet (ebenso KRU 15,97; 90,24); in KRU 22 aber, einem durch sein arab. Protokoll auf (nach) 759/60 datierter Papyrus, ist dreimal von $\Pi 2 \Pi$ NOY ФANOC die Rede (Z. 13, 15/16, 28/29). Ist Crums Ergänzung in Z. 6 also nicht unmöglich, so gibt es doch noch eine andere Möglichkeit, zu ergänzen: Parallel zu KRU 5,10ff. könnte auch hier die Adresse des Destinatärs ausgelassen worden sein, so daß sich an die Intitulatio unmittelbar Gruß und Homologie anschlössen, JYTE П५)HPE MПMAK[ (Z. 7) müßte dann der erste der Aussteller sein.

Zeile 8: Die bei Ciasca auf den Vatersnamen Geôrgios folgenden Buchstaben MTK[ können innerhalb der Aufzählung der Aussteller eigentlich nur entweder zu $<T P>M T K[\triangle C T P O N$ NХHME] oder als Anschluß $M<N>$ zum nächsten, mit $K[$ beginnenden Namen konjiziert werden. Es scheint leichter, $N$ als $\Pi z u$ verlesen, als die Zwei Buchstaben חP zu überlesen.

Zeile 9: Crums Emendation $\pi \epsilon<c>2 d$ l, '<ihr $>$ Gatte', schafft gegen das Faktum des sonst nicht erklärbaren betonten Artikels Abhilfe, Ciascas Lesung erklärt sich aus der von David mitunter verwendeten Ligatur C2, die bloßem 2 sehr ähnlich sieht.

Zeile 10: Die drei Buchstaben NAK[ bleiben unverständlich (auf den Namen muß die Filiation folgen), vielleicht sind sie aus MחM KK[ verlesen (obwohl Z. 19 der Vater des Papnoute nicht MAKdPIOC genannt wird)? Die Emendation חENTd-

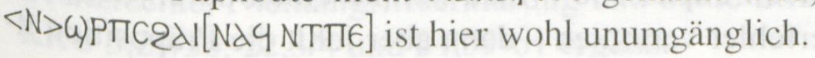


Zeile 11: Ciascas 2YKdION ist unverständlich, die mehrfach bezeugte Formel lautet

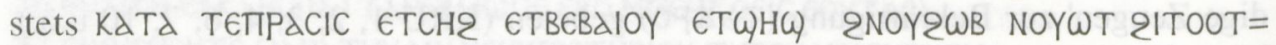
(cf. Steinwenter, Das Recht der koptischen Urkunden (Anm. 7), p. 52, n. 5; zu

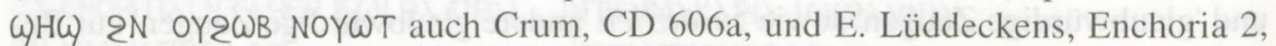
1972, p. 28f., n. 32). Die große Lücke zwischen 2N o[ und ] $\omega$ B für ganze zwei Buchstaben läßt hier wieder einen Zeilenwechsel vermuten.

Zeile 12: Willensfreiheitsklausel und Schwurformel (Z. 12-18) stimmen wörtlich mit den in KRU 5, 24, 90 und 98 belegten Formeln überein. Aus grammatischen Gründen ist der von Ciasca gelesene Relativsatz $€ T N O Y \omega G$ ) in Präs. II: $€ N O Y \omega G$ zu konjizieren.

Zeile 16: Es ist wahrscheinlich, daß Ciasca ПP0२€PHC€I analog zu ПP0२€PHCIC Z. 14/15 gelesen hat, während im Text חPOєIPHC€I stand: Die Schreiber aus Djême, die die Formel 'in jedem schönen und guten Vorsatz' verwendeten, unterschieden beim Gebrauch von $\pi \rho$ рохi im koptischen Kontext und dem deklinierten Wort in der griechisch zitierten Formel: so schreibt David in KRU 5, 24 und 98 ПPO२૯PHCIC, aber ПPOєIPHC€I; Aristophanes in KRU 27 TPO2dIPECIC, hingegen in KRU 11, 14, 15 und 87 ПPOЄPdICH. Überhaupt geben alle Belege in den KRU das ungebeugte Wort im koptischen Kontext mit 2 für spiritus asper wieder, während alle Belege für das deklinierte Wort in der griechischen Formel ohne 2 geschrieben sind.

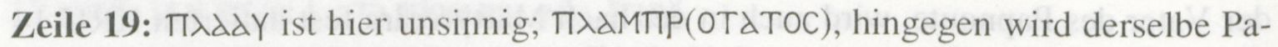
pnoute, Sohn des Stephanos, in KRU 22,28 angeredet.

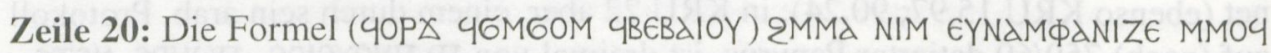
N2HT4 gehört an den Schluß der Urkunde; sowohl die eingeklammerten Ergänzungen als auch das Gelesene dürften von Ciasca mehr geraten worden sein. Diese Stelle läßt sich mit Davids Formular erklären, wo an der direkten Parallelstelle KRU 5,22 und drei vergleichbaren Nennungen des Destinatärs in KRU 22,17, 24,76 und 98,23 das sonst nur einmal in den $\operatorname{KRU}(7,37)$ bezeugte Verbum CYM $\Phi \Delta N I Z \epsilon$ in der synonym zu solchen Wendungen wie $\Pi € N T \Delta I G) P H \Delta H \lambda O Y$ MMOY NTT€ gebrauchten Form $\Pi / T-\epsilon N T \lambda I C Y M \Phi d N I Z \in M \Pi \epsilon 4 / C-P d N / M M O 4(N T \Pi \epsilon)$ Verwendung findet. Davon lassen sich in der so gründlich mißverstandenen Lesung Ciascas immerhin noch die Buchstaben ] ENT...C[ ] $\phi ৯ N I Z \in$ feststellen.

Zeile 22: Auf die Verkaufserklärung folgt nun die Verschreibung des Kaufobjektes, die mit $\Delta Y \omega$ ENC2dI NdK beginnt, cf. die Parallelstelle KRU 5,24ff., $\Delta Y \omega$ $€ 20 \gamma \mathrm{N}$ ist daher so zu emendieren.

Zeile 23: Meistens ist die Verschreibung des Kaufobjektes 'auf alle Zeiten, die kommen nach ...' auf die Personen des Ausstellers (MNNCWN, MNNCWI) oder des Destinatärs (MNNCWK, MNNCWTH) bezogen, doch ist mitunter (KRU 7,21; 12,32) auch die unpersönliche Variante MNNCWC belegt. Problematisch ist die Nennung des Kaufobjektes $\Pi \lambda H I$ bei Ciasca: Zunächst ist bei der Mehrzahl der Aussteller der Possesivartikel der 1. Person unpassend - man könnte einen durch Schematismus verursachten Schreiberfehler vermuten - dann aber fehlt die Präposition N- 
(M-) als Objektanschluß an das Verbum C2dI. Daher schlage ich die Emendation $<\mathrm{MMIH}>$ vor. Fraglich ist weiterhin, welche Posten verkauft wurden. In der Lücke nach $<M \Pi I H>[\ldots]$ THPप dürfte ein weiteres Kaufobjekt aufgeführt gewesen sein, welches den Ausstellern 'im (oder: vom) Haus unseres seligen Vaters Ammônius' 'gehört' hat $(\in T \omega) O O \Pi N \Delta N /=$ / häufig an dieser Stelle des Formulars: cf. KRU 1,51; $4,33 ; 5,26 ; 13,22 ; 23,15$; cf. auch $€ T A N 2 H K \in I \in P O I ~ 28,8)$. Unklar bleibt wegen der Lücken, ob das 'ganze' Haus mit dem des Ammônius identisch ist.

Zeile 24: Wären Ammônius und [Jô]hanna Eheleute (und Komeios Z. 1 ihr Sohn), wie Till, Datierung und Prosopographie (Anm. 2), p. 54, 59 und 121 mit Fragezeichen anheimstellte, wäre hier im Text, wenn schon nicht NEN€IOT€, so doch TENMAKAPIOC NEIWT AMMWNIOC MN[TEN]MAKAPId MMAY[ ]dNNA oder ähnliches zu erwarten gewesen. Nun bietet Ciascas Text aber П-, also wohl den Artikel eines zu Ammônius in Apposition stehenden Wortes, die beiden Buchstaben $\epsilon \mathrm{l}$ und eine Lücke. Da es weitere Hinweise im Text gibt, daß Ammônius nicht der Vater (da aber $\Pi \in N \in I \omega T$ genannt, wahrscheinlich der Großvater) der Aussteller ist, liegt es

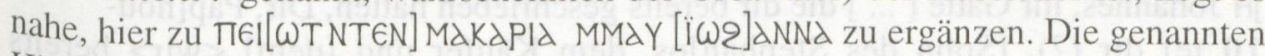
Hinweise sind:

1. Die Aussteller haben statt einer gemeinsamen Filiation NGHPE N- je ihre eigene Origo (Z. 1, 7, 8), wobei der Vater eines Ausstellers in Z. 8 Geôrgios heißt.

2. Die zum Haus gehörigen Gebrauchsgegenstände in Z. 28 werden bloß als 'auf uns seitens des Namens unserer seligen Mutter Jô[hann]a' gekommen bezeichnet.

Zeile 26: Die einzig in Frage kommende Lesung €ா€ৎTOYCd für Ciascas €OYTdCd hatte schon Crum in den Nachträgen zu KRU, p. 359, vorgeschlagen.

Zeile 27: Die häufig belegte Formel lautet ... MNNE4XPHCTHPION THPOY $€ T$ TN2HK€ICOdI $\in$ PO4. Die Schreibung XPICTHPION ist typisch für David, S. d. Psate $(5,28$;

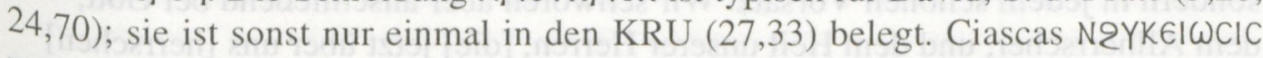
War schon von Crum in N2YK€IC $€ \epsilon$ emendiert worden. Die Endung $-\epsilon$ ist aber für dieses Verbum in den KRU nie belegt (fast immer - $-\lambda l$, selten $-\epsilon l$ ), und der Anschluß an XPICTHPION kann nur relativisch gewesen sein. David, S. d. Psate, pflegt $€ T \in N 2 Y K \in I C \theta d I$ zu schreiben (KRU 5,28; 24,70); die Buchstabenfolge ...N2YKEI... bei Ciasca macht diese Lesung am wahrscheinlichsten.

Zeile 28: Nur ein Buchstabe schließt die Lücke, die Ciasca mit vier Punkten taxierte - hier ist wieder ein Zeilenwechsel anzusetzen. Das merkwürdige Phänomen, daß nach ]NTENMAKAPId erst der Name und dann, korrekt mit Attributsanschluß zu MAKAPIA, MMAY folgt, ist, wenn nicht Ciascas Lesung, dann dem Schreiber als lax korrigierte Auslassung anzulasten.

Zeile 29: Der Text [ETEINAOYWN2 N4OECIC NAPXAION EBOX NAK EK TETPAГWNON ...], der nur ein Bruchteil des Zwischen Z. 29 und 30 fehlenden Textes (Nennung der Anrainer, Formel über den zuvor ausgehandelten Kaufpreis und seine Quittierung, Klausel über den Eigentumserwerb mit Aufzählung der verschiedenen Besitzrechte, Nichtangriffserklärung und beide Strafklauseln) ausmacht, ist nach KRU 5,29/30; 22,3/4 und 24,59-61 ergänzt. 
Zeile 29/30: Der Text in den Lücken ist nach den Stipulations- und Absolutionsklauseln von KRU 5,58-63; 19,76-89; 22,52-57; 24,116-123 und 90,17-21 ergänzt.

\section{Übersetzung}

- seine Mutt]er ist Anna - und Komeios, der Sohn des se[ligen ..., Einwohner von] Kastron

Djême, N[omos der Stadt Ermônt, wir veranlassen nach]her einen Namensfertiger, daß er unterschreibt für sie (sic statt 'uns'!), und glaubwürdige Zeugen, welche bezeugen werden diese Verkaufsurkunde, unübertretbar und 5 unerschütterlich durch (die) Gesetze, gemäß unserer eigenen Bitte und unserem Auftrag. [Gruß! Wir stimmen zu, wir ]ute, Sohn des sel[igen ..., Einwohner] von Djême, Nomos der S[tadt Ermônt, und ... ], der Sohn des seligen Geôrgios, <und> K[... ]MT€[ ? ]H Johannes, ihr Gatte [ ... ] die dir bereits geschrieben hatten, Dir, Pap[nou10 te] dir (?), [Sohn des Stephan]os, dem Käufer und [dem] <wir > bereits geschrieben hatten,

$<$ gemäß dieser $>$ Verkaufsurkunde, $<$ die $>$ schriftlich vorliegt, $<$ die $>$ stichhaltig ist, $<$ die $>$ in jeder Einzelheit ausgeglichen ist:

[Wir haben] uns dazu angeschickt, da wir dazu willens und überzeugt sind ohne irgendwelche

List oder Furcht oder Gewaltanwendung [oder Täuschung] oder irgendwelche Übervorteilung oder Betrüge-

rei, indem keinerlei Nötigung uns bedrückt - sondern durch unseren eigenen

15 Vorsatz - noch jedwede schlechte Gesinnung oder Arglist -,

sondern in jedem schönen Vorsatz. Wir schwören aber anschließend bei Gott, dem Allherrscher, und dem Heil unserer Herren, [die] jetzt über uns [herrschen] durch den Befehl Gottes. Wir verkau[fen d]ir [heu]te,

dir, <glänzendster> Pap[noutio]s, Sohn des Stephanos, dem Käufer und $\mathbf{2 0}<$ dessen Namen ich (sc. der Notar) $>$ kundgetan hatte, in

jeder gerechten Herrschaft nach vollständigem Verkaufsgesetz, und $<$ wir verschreiben $>$ [dir] vom heutigen Tag an auf alle Zeiten, die danach kommen, $<$ das $>$ ganze Haus $[\ldots]$, das liegt $[\ldots$ und ... was $]$ uns [gehört] im (oder: vom) Haus unseres seligen

Vaters Ammôniôs, des Va[ters unserer] seligen Mutter [Jôh]anna. Jenes ganze

25 Haus liegt im Kastron Djême südllich der Ge-

meindekirche, und du sollst sein Besitzer werden über $<$ die vier $>$ Seiten von seinen Fundamenten bis zum Luftraum samt all seinen Gebrauchsgegenständen, <die> dazugehören,

das, was auf mich ${ }^{\text {sic? }}$ gekommen ist seitens des [Namens] unserer seligen $<$ Mutter> Iô[hann]a

nach der Art, [wie ich jetzt seine ursprüngliche Lage nach den vier Seiten hin zeigen werde ... wohl sehr große Lücke!] diese Teilungs- und 
30 Auseinanderse[tzungsurkunde, ...] sie ist sicher, sie ist rechtskräftig bei [jeder] Gelegenheit, [da sie gezeigt

werden wird]. Wir wurden gefragt [und haben zugestimmt, man hat sie uns vorgelesen, wir haben sie bekräftigt durch Namensfertiger und Zeugen, wir haben sie verabschiedet nach Maßgabe der Gesetze ... ] klar daß [ ... ]

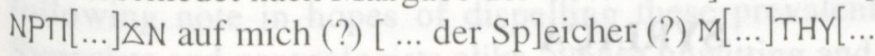

